\title{
Dramatic Performance by a Professional Actor for the Treatment of Patients with Behavioral and Psychological Symptoms of Dementia
}

\author{
Yusaku Maeda, ${ }^{1}$ Keita Fukushima, ${ }^{1}$ Satomi Kyoutani, ${ }^{1}$ James P. Butler, ${ }^{2,3,4}$ \\ Masahiko Fujii ${ }^{1}$ and Hidetada Sasaki ${ }^{1}$ \\ ${ }^{1}$ Sendai Tomizawa Hospital, Sendai, Miyagi, Japan \\ ${ }^{2}$ Department of Environmental Health, Harvard T.H. Chan School of Public Health, Boston, MA, USA \\ ${ }^{3}$ Division of Pulmonary and Critical Care Medicine, Brigham and Women's Hospital, Boston, MA, USA \\ ${ }^{4}$ Harvard Medical School, Boston, MA, USA
}

\begin{abstract}
Behavioral and psychological symptoms of dementia (BPSD) challenge caregivers, leading to caregiver burden and subsequent nursing home or inpatient placement in a psychiatric hospital for dementia. Favorable positive emotions should be an important goal for the treatment of negative emotions of BPSD. Arts are one of the most profound areas to stimulate favorable emotions. We have asked a professional actor, who was not involved in the daily care and regular rehabilitations, to give a dramatic performance by reading selected stories as if the patients with BPSD felt to be in the audiences of a theater. We wondered whether a dramatic performance by the actor might be a way to respond to the complex needs of inpatients with BPSD, especially focused on favorable emotions. New inpatients (Alzheimer's disease, vascular dementia, or dementia with Lewy bodies) were randomly assigned to a control group $(n=20)$ and a dramatic performance group $(n=14)$ in Sendai Tomizawa Hospital, a psychiatric hospital for dementia, in Japan. Dramatic performances were performed for one and half hours once per week for 3 months. Neuropsychiatric Inventory for BPSD decreased in both groups and delightful emotional index (DEI) for favorable emotions increased in the intervention group but not in the control group after 3 months. At 3 months, there was an increase of DEI in intervention group compared with control group. We conclude that dramatic performance may be one of the appropriate interventions in patients with BPSD, as it appears to help in their favorable emotional state.
\end{abstract}

Keywords: behavioral and psychological symptoms of dementia; delightful emotional index; dramatic performance; favorable emotions; negative emotions

Tohoku J. Exp. Med., 2020 November, 252 (3), 263-267.

\section{Introduction}

Dementia patients may exhibit behavior such as agitation, anger, and aberrant motor activity that challenges caregivers, leading to caregiver burden and subsequent placement in a nursing home or a psychiatric hospital for patients with dementia in severe cases. We have suggested that favorable positive emotions should be an important goal for the treatment of negative emotions of behavioral psychological symptoms of dementia (BPSD) (Fujii et al. 2019). In contrast to negative emotional status of BPSD, positive emotional status such as exchanging greetings appropriately, exhibiting facial expressiveness, showing interested in something, carrying on a conversation, and showing delight in something are defined as favorable emotions, which is assessed with the Delightful Emotional Index (DEI) (Fujii et al. 2019). Arts are one of the most profound areas to stimulate favorable emotions. We asked an actor to give a dramatic performance by reading selected stories as if the patients were in the audience of a theater. We wondered if these dramatic performances for patients with BPSD might be a way to respond to patient's complex needs, especially focused on favorable emotions.

Dramatic performance has been played for the last 10 years by a professional actor, who played an active part in Sendai city for patients with BPSD. At the beginning the

Received April 14, 2020; revised and accepted October 16, 2020. Published online November 10, 2020; doi: 10.1620/tjem.252.263. Correspondence: Hidetada Sasaki, Sendai Tomizawa Hospital, 11-4 Terashiro, Tomizawa, Taihaku-ku, Sendai, Miyagi 982-0032, Japan. e-mail: hsasakihide@yahoo.co.jp

(C)2020 Tohoku University Medical Press. This is an open-access article distributed under the terms of the Creative Commons Attribution-NonCommercial-NoDerivatives 4.0 International License (CC-BY-NC-ND 4.0). Anyone may download, reuse, copy, reprint, or distribute the article without modifications or adaptations for non-profit purposes if they cite the original authors and source properly.

https://creativecommons.org/licenses/by-nc-nd/4.0/ 
actor was dismissed by many of the patients, some of whom left in the middle of the performance. The actor responded by trial and error to play dramatic performances by asking the patients for feedback, such as for keywords to stimulate favorable emotions of the patients, and searching materials to read including books which were deemed appropriate for the patients, and preparing narration how to inspire the patients through the reading contents. Dramatic performances have been gradually accepted by the patients and lengths of reading the stories were gradually increased from short ones of about $10 \mathrm{~min}$ to longer ones up to $50 \mathrm{~min}$. So far, nonpharmachological interventions for BPSD have been focused only on negative emotions such as violence, ugly perceptions, and antisocial behavior, but interventions for favorable emotions in dementia patients have not been reported (Luk 2017; The Lancet Neurology 2018). We have suggested that dramatic performance might be effective in stimulating emotional functions assessed with MiniEmotional State Examination (MESE) (Fujii et al. 2014a) in patients with dementia (Maeda et al. 2015). The MESE explicitly probes organic feeling of comfort or discomfort in a variety of settings, including those associated with the human condition, moral sense, social sense, feelings of happiness and sadness. We have reported that dramatic performance showed the highest points of Emotional Satisfaction Index (ESI) among various recreational programs (Maeda et al. 2016). The ESI is measured by summation of positive and negative satisfactory reactions of patients during performing recreational programs, including staying awake, falling asleep, participating or ignoring the activity, showing interest or rejection, and so on. In the present study, we adopted dramatic performances for patients with BPSD to examine whether these dramatic performances improved favorable emotions assessed with DEI and relieved BPSD.

\section{Subjects and Methods}

Dramatic performances consisted of narration and talks by a professional actor, Mr. Maeda, Y., who was not involved in daily care or regular rehabilitation activities. A variety of themes were covered, such as love, happiness, and sadness, which were presented like a theatre performance and were followed by free discussion. Themes were special stories during and after World War II, stories from novels, and comic stories including "Rakugo" in Japanese, which took from 20 to $40 \mathrm{~min}$ for one story. During the course, the actor moved quickly to the next theme if the participants lost interest. Many other supplementary materials were used such as slides, videos, or drawing pictures of the stories. Dramatic performances were played every Friday afternoon for one and half hours for about 10 inpatients in a small room of Sendai Tomizawa Hospital in Japan, a psychiatric hospital for dementia, from September 2018 to April 2019. Patients with Alzheimer's disease, vascular dementia, or dementia with Lewy bodies were included, exclusion criteria included patients with other types of dementia such as frontotemporal lobar degenera- tion, corticobasal degeneration, and dementia with psychiatric disorders such as brain injury, alcoholism, and schizophrenia and patients who were hard of hearing, weak sighted, or bedridden. The diagnosis of dementia was made for a total of 46 new inpatients according to the Diagnostic and Statistical Manual of Mental Disorder, Fifth Edition (DSM-5) criteria (American Psychiatric Association 2018).

Forty-six new inpatients were randomly assigned in a $3: 2$ ratio by a computerized random number function in Microsoft Excel to a control group (usual care without dramatic performance, $\mathrm{n}=26$ ) or the intervention group (usual care with dramatic performances, $n=19$ ). Characteristics of patients were assessed during the week-day except Friday by nurses who were blinded in the present study using Barthel Index (Mahoney and Barthel 1965). This metric ranged from $0-100$, higher scores indicate better performance for ADL. We also used Mini-Mental State Examination (MMSE) (Folstein et al. 1975), which ranges from 0-30, higher scores indicate better performance for cognitive function, Neuropsychiatric Inventory (NPI) (Cummings et al. 1994) ranged 0-108, lower scores indicate better performance for BPSD, and Delightful Emotional Index (DEI) (Fujii et al. 2019) ranged 0-90, higher scores for better performance for favorable emotions at baseline and three months after hospitalization. Patients with MMSE scores $\leqq 10$ points at baseline were not included for the present study. Psychotropics were prescribed for new inpatients with BPSD by a psychiatrist, who was blinded to the protocol. Prescribed one tablet and/or pack of drugs were as follows; Antipsychotics; chlorpromazine $(12.5 \mathrm{mg})$, levomepromazine $(5 \mathrm{mg})$, risperidone $(1 \mathrm{mg})$, olanzapine $(5 \mathrm{mg})$, quetiapine fumarate $(25 \mathrm{mg})$; Nonantipsychotics; etizolam (1 mg), diazepam ( $2 \mathrm{mg})$, trazodone hydrochloride $(25 \mathrm{mg})$, sodium valproate $(200 \mathrm{mg})$, phenytoin $(100 \mathrm{mg})$, tiapride hydrochloride $(25 \mathrm{mg})$, YuiGan San (2.5 mg); Antidementia drugs; donepezil (5 mg), rivastigmine $(9 \mathrm{mg})$, galantamine hydrochloride $(8 \mathrm{mg})$, memantine hydrochloride $(20 \mathrm{mg})$. The study was approved by the ethics committee of Yamagata Kousei Hospital, one of the partnerships of management for Sendai Tomizawa Hospital (approval number 2018-7). Statistics were calculated by $t$-test and Mann-Whitney $U$-test using BellCurve for Excel. Data are shown as mean \pm SD.

\section{Results}

In the control group, 6 patients dropped out because of infections or other causes, and in the intervention group, 5 patients dropped out because of infections, loss of interests, or miscellaneous reasons. Finally 20 patients (Alzheimer's disease: $\mathrm{n}=15$, vascular dementia: $\mathrm{n}=2$, dementia with Lewy bodies: $\mathrm{n}=3$ ) in control group (ages: $82 \pm 9$ years, $\mathrm{f} /$ $\mathrm{m}: 10 / 10$ patients) and 14 patients (Alzheimer's disease: $\mathrm{n}$ $=12$, vascular dementia: $\mathrm{n}=1$, dementia with Lewy bodies: $\mathrm{n}=1$ ) in intervention group (ages: $84 \pm 6$ years, $\mathrm{f} / \mathrm{m}: 6 / 8$ patients) were successfully followed for 3 months. Changes of characteristics and drugs of control and intervention 
groups are shown in Table 1. NPI showed significant decrease in both control and intervention groups. On the NPI subscales, significant improvements in hallucinations, agitation/aggression, irritability/anger, aberrant motor activity, and nighttime disturbance were observed in both control and intervention groups (Table 2). Delusions were signifi-

Table 1. Characteristics and drugs of control and dramatic performance groups at baseline and 3 months.

\begin{tabular}{lcc}
\hline & Baseline & 3 months \\
\hline Control group & & \\
Barthel Index $(\mathrm{n}=20)$ & $54 \pm 21$ & $51 \pm 26$ \\
MMSE $(\mathrm{n}=20)$ & $15 \pm 3$ & $15 \pm 7$ \\
NPI $(\mathrm{n}=20)$ & $40 \pm 22$ & $24 \pm 17^{* * *}$ \\
DEI $(\mathrm{n}=20)$ & $19 \pm 15$ & $30 \pm 16$ \\
Antipsychotics $(\mathrm{n}=12)$ & $2.0 \pm 1.7$ & $3.6 \pm 3.6$ \\
Nonantipsychotics $(\mathrm{n}=9)$ & $1.3 \pm 1.3$ & $0.9 \pm 1.4$ \\
Antidementia drugs $(\mathrm{n}=6)$ & $0.8 \pm 0.7$ & $1.1 \pm 0.7$ \\
Intervention group & & \\
Barthel Index $(\mathrm{n}=14)$ & $60 \pm 30$ & $63 \pm 34$ \\
MMSE (n=14) & $16 \pm 6$ & $15 \pm 8$ \\
NPI (n=14) & $51 \pm 19$ & $14 \pm 8^{* * *}$ \\
DEI $(\mathrm{n}=14)$ & $15 \pm 17$ & $51 \pm 16^{* * *}, \# *$ \\
Antipsychotics $(\mathrm{n}=7)$ & $2.4 \pm 2.4$ & $0.5 \pm 0.8$ \\
Nonantipsychotics $(\mathrm{n}=12)$ & $1.1 \pm 1.6$ & $2.4 \pm 2.4$ \\
Antidementia drugs $(\mathrm{n}=4)$ & $1.3 \pm 0.9$ & $0.7 \pm 0.5$ \\
\hline
\end{tabular}

Numbers of drugs are summation of tablets and/or packs per day.

MMSE, mini-mental state examination; NPI, neuropsychiatric intervention; DEI, delightful emotional index.

${ }^{* * *} \mathrm{p}<0.001$ between baseline and 3 months after hospitalization (paired $t$-test).

${ }^{\#} \mathrm{p}<0.05$ between Barthel Index, MMSE, NPI, or DEI of control and intervention groups (the differences between baseline and 3 months of each index of control and intervention groups are compared using Mann-Whitney $U$-test). cantly improved in the intervention group. DEI showed significant increase in the intervention group but not in the control group. At 3 months, there was an increase of DEI in intervention group compared with controls. Antipsychotics showed a tendency to increase in the control group and decrease in the intervention group but there is no equivalent conversion for antipsychotics including the other drugs and comparisons of drugs were not performed.

\section{Discussion}

In the present study, dramatic performances by the professional actor caused deep sympathy, increased favorable emotions, and improved negative emotions of BPSD in the intervention group. NPI showed significant decrease in both intervention and control groups most likely because of treatments with psychotropics and antidementia drugs and nondrug therapy. However, favorable emotions did not significantly increase with regular recreation and rehabilitation in the control group, probably due to insufficiency of favorable emotional stimulations. This was the motivation for trying the present study of dramatic performances, in which we found significant improvement in favorable emotional function compared with the controls. Effective nonpharmacological treatment is heavily dependent on adequate manpower and caregiver training (DeYoung et al. 2002; Nakanishi et al. 2018). The manpower for care of the patients with BPSD is often limited and caregivers are exhausted. We asked the professional actor staying near the hospital to support nonpharmacological treatment by playing dramatic performance once a week, which is a kind of social prescription to take advantage of the power of the arts. The actor himself extends the activity as one of the stages of performances as well as regular theatrical activities. Client-centered and tailored therapy guided by the patient's background, interest, culture, religion, and life factors is the most important concept when treating BPSD (Eggenberger et al. 2013). Since BPSD is so complex and

Table 2. NPI subscale scores of control and dramatic performance groups at baseline and 3 months.

\begin{tabular}{llllll}
\hline \multirow{2}{*}{ NPI subscales } & \multicolumn{2}{c}{ Control group $(\mathrm{n}=20)$} & & \multicolumn{2}{c}{ Intervention group $(\mathrm{n}=14)$} \\
\cline { 2 - 3 } \cline { 5 - 6 } Delusions & Baseline & 3 months & & Baseline & 3 months \\
Hallucinations & $2.1 \pm 3.2$ & $1.7 \pm 2.7$ & & $3.8 \pm 3.1$ & $1.1 \pm 1.9^{*}$ \\
Agitation/aggression & $3.7 \pm 3.9$ & $1.8 \pm 2.3^{*}$ & & $4.9 \pm 4.2$ & $0.9 \pm 1.6^{*}$ \\
Dysphonia & $4.6 \pm 3.7$ & $2.3 \pm 2.7^{*}$ & & $5.8 \pm 4.7$ & $1.5 \pm 3.1^{*}$ \\
Anxiety & $2.1 \pm 2.6$ & $1.9 \pm 2.1$ & & $2.9 \pm 2.3$ & $0.6 \pm 1.5$ \\
Euphonia & $3.9 \pm 4.8$ & $2.8 \pm 2.9$ & & $4.7 \pm 4.8$ & $1.2 \pm 3.1$ \\
Apathy & $1.4 \pm 2.7$ & $1.1 \pm 1.9$ & & $1.2 \pm 1.9$ & $0.7 \pm 0.9$ \\
Disinhibition & $3.6 \pm 4.1$ & $3.1 \pm 3.7$ & & $2.9 \pm 2.8$ & $0.8 \pm 1.5$ \\
Irritability/anger & $2.6 \pm 3.7$ & $1.5 \pm 2.8$ & & $3.2 \pm 3.2$ & $1.7 \pm 2.1$ \\
Aberrant motor activity & $5.8 \pm 5.1$ & $2.7 \pm 3.4^{*}$ & & $6.9 \pm 4.4$ & $1.8 \pm 2.9^{*}$ \\
Night-time disturbance & $4.1 \pm 5.2$ & $2.0 \pm 2.7^{*}$ & & $5.6 \pm 5.1$ & $2.0 \pm 3.2^{*}$ \\
Eating disturbance & $4.8 \pm 4.2$ & $1.7 \pm 1.6^{*}$ & & $6.5 \pm 4.1$ & $1.6 \pm 1.9^{*}$ \\
\cline { 1 - 2 } & $1.4 \pm 2.3$ & $1.1 \pm 2.4$ & & $2.1 \pm 2.2$ & $0.5 \pm 1.9$ \\
\hline
\end{tabular}

${ }^{*} \mathrm{p}<0.05$ between subscale scores at baseline and 3 months (paired $t$-test). 
hard to improve with usual treatments, finding others, outside the hospital, to contribute to patient care through the arts, would be one of the important alternative ways to both help the patients and to lighten the burden on caregivers.

During dramatic performances many patients showed deep emotional states and favorable emotions were increased; we have observed choking with tears in some patients. Even after hearing a tragic wartime story, one of the patients sympathized, and the sympathy spread through the group, and it eventually resonated with everyone, with patients in a sense liberated from stresses such as anxiety and fear. So far, the common non-pharmacological managements for BPSD, such as exercise therapy, reminiscence therapy, music therapy, sensory therapy, aromatherapy, and so on, have focused on to deceasing negative emotions assessed through e.g., NPI, (Ijaopo 2017) but we need to pay equal attention to increases in favorable emotions as well as decreases in negative emotions (Nietzsche 2005), assessed e.g., with DEI. Favorable emotions might be the other treatable emotions besides negative emotions for patients of BPSD. The presence of BPSD suggests the relative preservation of the emotional function generated from the limbic system compared to cognitive function generated from the neocortex (Butler et al. 2012; Fujii et al. 2014b, 2018). Negative emotions like BPSD could be in part relieved with psychotropics but favorable emotions are not, as yet, treatable pharmacologically. The feeling of appreciation in dementia patients, which is often associated with positive human interaction, is far superior to that of infants, even though their cognitive functions might indicate infantile cognitive levels. Some dementia patients with such positive human interaction might decline to participate in more traditional regular rehabilitative activities, such as playing simple games, drawing and TV (Maeda et al. 2016).

Although nonpharmacological management is the first and preferred option for BPSD, pharmacotherapy is a second-line option for more severe and persistent BPSD not responding to nonpharmacological measures (American Psychiatric Association 2017). In the present study pharmacological treatments were prescribed in some patients and antipsychotics showed a tendency to increase in the control group, probably in order to treat BPSD, and showed a tendency to decrease in the intervention group, but statistical calculation for drugs were not performed because of unknown equivalences among drugs. However, improvements of favorable emotions as well as negative emotions might be an indication for withdrawing antipsychotics in the intervention group. Antipsychotics are suggested to reduce favorable emotions as well as BPSD, and this should be considered in prescribing medications for patients with dementia (Fujii et al. 2019). A tendency of increased antipsychotics might be another reason for not finding increased favorable emotions in the control group. The adverse side effects of antipsychotics are well known to cause extra medical expenses (Hurd et al. 2013) besides reductions of brain functions and ADL (Azumi et al. 2011). The present expense to pay the actor as a volunteer might be far cheaper and healthful than prescription of antipsychotics in terms not only for a countermeasure for polypharmacy and drug cost but also cost for adverse side effects (Gill et al. 2007).

There are some limitations of the present study. First, the patients with MMSE $\leqq 10$ were omitted from the study since patients with advanced dementia of cognitive function could not follow the stories. Second, character and favorable nature of emotions of each patient are different depending on their life history and taste and some patients dropped out because of loss of interest and a smaller number of patients were enrolled in the intervention group. But the ratio of drop-out of both groups were virtually the same ( 0.23 for control group and 0.26 for intervention group). Third, affinity of characteristic of the actor for the patients with BPSD is necessary for dramatic performance because tender care with respect and open mind for the patients with BPSD as well as emotional performances were required. At the beginning we interviewed the professional actor, Mr. Maeda, Y., to see whether he was a suitable actor to serve the patients with BPSD or not. Mr. Maeda tenderly cared for the patients with respect and an open mind throughout dramatic performances. The doctors including psychiatrist, nurses, caregivers, and occupational therapists practiced daily cares and regular rehabilitations in both control and intervention groups equally and Mr. Maeda was not involved in the daily care and regular rehabilitations and thus would not confound the beneficial effects we found with dramatic performances. Finally, distributions of diagnosis of the dementia patients were similar between control and intervention groups and characteristics of Barthel Index, MMSE, NPI, and DEI at baseline were not significantly different between two groups and as above, would have little confounding effects of disease-related differences on the results. We conclude that dramatic performances, one among many of the arts, may be an appropriate intervention in patients with BPSD, who are caring of humanity in their deep mind, as it appears to help in their deep favorable emotional state.

\section{Conflict of Interest}

The authors declare no conflict of interest.

\section{References}

American Psychiatric Association (2017) Practice Guideline on the Use of Antipsychotics to Treat Agitation or Psychosis in Patients with Dementia, American Psychiatric Association Publishing, Washington, DC, USA.

American Psychiatric Association (2018) Diagnostic and Statistical Manual of Mental Disorders, Fifth Edition: DSM-5, American Psychiatric Association Publishing, Washington, DC, USA.

Azumi, M., Ishizuka, S., Fujii, M. \& Sasaki, H. (2011) Antipsychotics and cognitive function. Psychogeriatrics, 11, 79-82.

Butler, J.P., Fujii, M. \& Sasaki, H. (2012) New lessons of nurturing life for geriatric patients. Tohoku J. Exp. Med., 227, 203-210.

Cummings, J.L., Mega, M., Gray, K., Rosenberg-Thompson, S., Carusi, D.A. \& Gornbein, J. (1994) The Neuropsychiatric 
Inventory: comprehensive assessment of psychopathology in dementia. Neurology, 44, 2308-2314.

DeYoung, S., Just, G. \& Harrison, R. (2002) Decreasing aggressive, agitated, or disruptive behavior: perticipation in a behavior management unit. J. Gerontol. Nurs., 28, 22-31.

Eggenberger, E., Heimerl, K. \& Bennett, M.I. (2013) Communication skills training in dementia care: a systematic review of effectiveness, training content, and didactic methods in different care settings. Int. Psychogeriatr., 25, 345-358.

Folstein, M.F., Folstein, S.E. \& McHugh, P.R. (1975) "Minimental state". A practical method for grading the cognitive state of patients for the clinician. J. Psychiatr. Res., 12, 189-198.

Fujii, M., Butler, J.P., Hirazakura, A. \& Sasaki, H. (2014a) MiniEmotional State Examination for dementia patients. Geriatr. Gerontol. Int., 14, 508-513.

Fujii, M., Butler, J.P. \& Sasaki, H. (2014b) Emotional function in dementia patients. Psychogeriatrics, 14, 202-209.

Fujii, M., Butler, J.P. \& Sasaki, H. (2018) Core symptoms and peripheral symptoms of dementia. Geriatr. Gerontol. Int., 18, 979-980.

Fujii, M., Butler, J.P. \& Sasaki, H. (2019) Antipsychotic drug use and favourable natures of emotional functions in patients with dementia. Psychogeriatrics, 19, 320-324.

Gill, S.S., Bronskill, S.E., Normand, S.L., Anderson, G.M., Sykora, K., Lam, K., Bell, C.M., Lee, P.E., Fischer, H.D., Herrmann, N., Gurwitz, J.H. \& Rochon, P.A. (2007) Antipsychotic drug use and mortality in older adults with dementia. Ann. Intern.
Med., 146, 775-786.

Hurd, M.D., Martorell, P., Delavande, A., Mullen, K.J. \& Langa, K.M. (2013) Monetary costs of dementia in the United States. N. Engl. J. Med., 368, 1326-1334.

Ijaopo, E.O. (2017) Dementia-related agitation: a review of nonpharmacological interventions and analysis of risks and benefits of pharmacotherapy. Transl. Psychiatry, 7, e1250.

Luk, J.K.H. (2017) Pharmacological management of behavioural and psychological symptoms of dementia. Asian J. Gerontol. Geriatr., 12, 65-68.

Maeda, Y., Kaneda, E., Fujii, M. \& Sasaki, H. (2016) Emotional satisfaction index for dementia patients. Geriatr. Gerontol. Int., 16, 530-532.

Maeda, Y., Kaneda, E., Hasegawa, S., Yoshida, S., Fujii, M. \& Sasaki, H. (2015) Dramatic emotional therapy for dementia patients. Psychogeriatrics, 15, 277-278.

Mahoney, F.I. \& Barthel, D.W. (1965) Functional evaluation: the Barthel Index. Md. State Med. J., 14, 61-65.

Nakanishi, M., Endo, K., Hirooka, K., Granvik, E., Minthon, L., Nägga, K. \& Nishida, A. (2018) Psychosocial behaviour management programme for home-dwelling people with dementia: a cluster-randomized controlled trial. Int. J. Geriatr. Psychiatry, 33, 495-503.

Nietzsche, F. (2005) Also sprach Zarathustra: Ein Buch für Alle und Keinen, Anaconda Verlag GmbH, Köln, Germany.

The Lancet Neurology (2018) Response to the growing dementia burden must be faster. Lancet Neurol., 17, 651. 